\title{
Charm-strange meson production in ultrarelativistic heavy-ion collisions at energies available at the CERN Large Hadron Collider
}

\author{
Shuang $\mathrm{Li}^{1,2, *}$ and Chaowen Wang ${ }^{1, \dagger}$ \\ ${ }^{1}$ College of Science, China Three Gorges University, Yichang 443002, China \\ ${ }^{2}$ Key Laboratory of Quark and Lepton Physics (MOE), Central China Normal University, Wuhan 430079, China
}

(Received 15 May 2018; revised manuscript received 14 August 2018; published 20 September 2018)

\begin{abstract}
The nuclear modification factor $R_{A A}$ and the elliptic flow coefficient $v_{2}$ of charm-strange meson $D_{s}^{+}$is systematically studied in $\mathrm{Pb}-\mathrm{Pb}$ collisions at $\sqrt{s_{N N}}=5.02$ and $2.76 \mathrm{TeV}$. During the modeling, the coupling strength between the injected charm quark and the incident medium constituents is extracted from the lattice QCD calculations: $2 \pi T D_{s}=$ const (Model-A) and $2 \pi T D_{s}=1.3+\left(T / T_{c}\right)^{2}$ (Model-B). We find that, comparing $R_{A A}\left(D_{s}^{+}\right)$with $R_{A A}$ (nonstrange), the heavy-light coalescence effect is more pronounced for the former one, resulting in an enhancement behavior in the range $2 \lesssim p_{T} \lesssim 5 \mathrm{GeV}$. The predictions for $R_{A A}\left(D_{s}^{+}\right)$and $R_{A A}$ (nonstrange) favor Model-A to have a better description of the measured $p_{T}$ dependence at both energies, while the predictions for $v_{2}$ prefer Model-B at moderate $p_{T}\left(2 \lesssim p_{T} \lesssim 4 \mathrm{GeV}\right)$. Therefore, it is necessary to consider the temperature and/or momentum dependence of $2 \pi T D_{s}$ to describe simultaneously $R_{A A}\left(D_{s}^{+}\right)$and $v_{2}\left(D_{s}^{+}\right)$in different centrality classes in $\mathrm{Pb}-\mathrm{Pb}$ collisions.
\end{abstract}

DOI: 10.1103/PhysRevC.98.034914

\section{INTRODUCTION}

Ultrarelativistic heavy-ion collisions provide a unique opportunity to produce and study the properties of strongly interacting matter within an extreme high temperature and energy density environment, where a phase transition is expected from the ordinary hadron state to its deconfined constituents, namely a quark-gluon plasma (QGP) [1,2]. Heavy quarks (HQs) such as the charm and bottom are of particular interest among the various probes of the QGP [3-5]. Due to their large mass, they are mainly produced at the early stage of collisions via the hard scattering process, and subsequently interact with the QGP constituents without affecting their mass, resulting in negligible regeneration propagating through the medium. Meanwhile, the HQ flavor is conserved during the interaction with QGP constituents; therefore, the initially produced HQ will experience the full evolution of the hot and dense medium.

While traversing the QGP medium, a heavy quark will interact with the medium constituents and thus lose part of its initial energy via both elastic $(2 \rightarrow 2$, collisional processes [6]) and inelastic scatterings $(2 \rightarrow 2+X$, including gluon radiation [7]), named the collisional and radiative energy losses, respectively. The energy loss effect together with

\footnotetext{
*1ish@ctgu.edu.cn

†'wangchaowen@ctgu.edu.cn
}

Published by the American Physical Society under the terms of the Creative Commons Attribution 4.0 International license. Further distribution of this work must maintain attribution to the author $(s)$ and the published article's title, journal citation, and DOI. Funded by $S C O A P^{3}$. the HQ hadronization mechanisms can be investigated by measuring the nuclear modification factor

$$
R_{A A}\left(p_{T}, y\right)=\frac{d^{2} \sigma_{A A} / d p_{T} d y}{d^{2} \sigma_{p p} / d p_{T} d y}
$$

of the final heavy-flavor productions such as those of open charmed mesons (i.e., $D$ mesons including $D^{0}, D^{+}, D^{*+}$, and $\left.D_{s}^{+}[8]\right)$, where $d^{2} \sigma_{A A} / d p_{T} d y$ is the $p_{T}$ and $y$ doubledifferential production cross section in nucleus-nucleus collisions, scaled by the number of binary nucleon-nucleon collisions; $d^{2} \sigma_{p p} / d p_{T} d y$ is the double-differential result in nucleon-nucleon collisions. The deviation of $R_{A A}$ from unity is sensitive to the nuclear effects, e.g., the initial (anti)shadowing and the subsequent in-medium energy loss. In addition, the elliptic flow coefficient

$$
v_{2}=\left\langle\frac{p_{x}^{2}-p_{y}^{2}}{p_{x}^{2}+p_{y}^{2}}\right\rangle
$$

allows one to describe the anisotropy of the transverse momentum, hence $v_{2}$ is sensitive to the equation of state (EoS) and initial conditions in the low- $p_{T}$ region, and it is also able to reflect path-length dependence of the energy loss at high $p_{T}$.

Many models were developed [9-14] to study the comprehensive sets of the available measurements of nonstrange charmed mesons, e.g., $D^{0}, D^{+}$, and $D^{*+}$. It was realized [15-18] that the simultaneous description of their $R_{A A}$ and $v_{2}$ requires further understanding of the temperature dependence of the coupling strength $\left(2 \pi T D_{s}\right)$ between the injected (heavy) quark and the incident medium constituent. The charm-strange meson $D_{s}^{+}(c \bar{s})$ production is more interesting with respect to nonstrange charmed mesons, since its valence quark content consists of charm and (anti)strange 
quarks, which will couple the well-known strangeness enhancement [19]. $D_{s}^{+}$spectra will be therefore affected by both the charm conservation and the strangeness enhancement effects in heavy-ion collisions. However, few models [20] were dedicated to investigating the $D_{s}^{+}$meson spectra, as well as its $R_{A A}$ and $v_{2}$, until now.

Based on the previous work, we try to address this question by taking into account the various temperature dependences of $2 \pi T D_{s}$ which are phenomenologically extracted from the lattice QCD calculation, and then investigate their effects on the observables $\left(R_{A A}\right.$ and $\left.v_{2}\right)$, in particular for the charm-strange meson $D_{s}^{+}$at energies available at the CERN Large Hadron Collider (LHC). Meanwhile, as pointed in Ref. [21], we will explore the propagation of theoretical uncertainties in energyloss predictions, for instance the $p p$ baseline calculation and the (anti)shadowing parametrization, in this analysis.

This paper proceeds as follows: Section II is dedicated to introducing the general steps of our hybrid model, including the initial condition, hydrodynamics expansion of the fireball, heavy quark Brownian motion, and the subsequent hadronization processes. Section III presents the results such as the production cross section and $R_{A A}$ and $v_{2}$ of $D_{s}^{+}$mesons in $p p$ and $\mathrm{Pb}-\mathrm{Pb}$ collisions. A comparison with available measurements is performed as well. Section IV contains the summary and conclusion.

\section{METHODOLOGY}

We construct a theoretical framework [22] to study the charm quark evolution in ultrarelativistic heavy-ion collisions. The general steps are outlined as follows, including the estimation of the theoretical uncertainties.

\section{A. Hybrid model construction}

\section{Initial conditions for the hydrodynamical evolution}

The initial spatial distribution of heavy quark pairs is sampled according to the initial entropy density distributions. The relevant transverse profile is modeled by a Glauber-based approach [23], while the longitudinal profile is described by a data-inspired phenomenological function [22]. The initial momentum distribution of heavy quark pairs is obtained via FONLL calculations [24-26]. Finally, the $c \bar{c}$ are generated back to back $(\Delta \phi=\pi)$ before including the nuclear shadowing effect [27].

The initial entropy density distributions will be taken as inputs of the subsequent hydrodynamical evolution, which can be described by utilizing a 3+1-dimensional relativistic viscous hydrodynamics model [28] with the start time scale $\tau_{0}=0.6 \mathrm{fm} / c$ and the shear viscosity $\eta / s=1 /(4 \pi)$. The tuning parameters in these modules are determined by a model-to-data comparison [22].

\section{Heavy quark diffusion}

The Brownian motion of a charm quark when propagating through the quark-gluon plasma (QGP), is described by utilizing the Langevin transport equation, and it can be modified to incorporate both the collisional and radiative energy loss processes [11]:

$$
d p=\left(F^{\text {Drag }}+F^{\text {Diff }}+F^{\text {Gluon }}\right) d t,
$$

with the drag force

$$
F^{\text {Drag }}=-\Gamma(p) p,
$$

the thermal random force ${ }^{1}$

$$
\left\langle F_{i}^{\text {Diff }}(t) F_{j}^{\text {Diff }}(t+n \Delta t)\right\rangle \equiv \frac{\kappa(p)}{\Delta t} \delta_{i j} \delta_{0 n},
$$

and the recoil force

$$
F^{\text {Gluon }}=-\frac{d p^{\text {Gluon }}}{d t} .
$$

$p^{\text {Gluon }}$ indicates the momentum of the radiated gluon, which can be quantified by the pQCD higher-twist calculation [29]. It is assumed [11] that the fluctuation-dissipation relation is still validated between the drag [Eq. (4)] and the diffusion terms [Eq. (5)] in Eq. (3):

$$
\Gamma(p)=\frac{\kappa(p)}{2 T E},
$$

where $\Gamma(p)$ and $\kappa(p)$ denote the drag and the momentum diffusion coefficients, respectively, and they can be rewritten via the spatial diffusion coefficient $2 \pi T D_{s}$ [30],

$$
\begin{gathered}
\Gamma=\frac{1}{\left(2 \pi T D_{s}\right)} \frac{2 \pi T^{2}}{E}, \\
\kappa=\frac{1}{\left(2 \pi T D_{s}\right)} 4 \pi T^{3} .
\end{gathered}
$$

Note that the definition of $2 \pi T D_{s}$ is extended from the zeromomentum region to a larger momentum region. As discussed in Ref. [22], $2 \pi T D_{s}$ can be obtained by performing a phenomenological fit analysis with the lattice QCD calculations. Two approaches are summarized as follows:

Model-A

$$
2 \pi T D_{s}=7 .
$$

In this approach the drag coefficient behaves as $\Gamma \propto T^{2}$, which is similar to the anti-de Sitter-space/conformalfield-theory (AdS/CFT) or pQCD calculation [9].

Model-B

$$
2 \pi T D_{s}=1.3+\left(\frac{T}{T_{c}}\right)^{2},
$$

where $T_{c}$ denotes the critical temperature. In this approach the drag coefficient behaves with a weak $T$ dependence, which is consistent with the results shown in Ref. [17,31].

Figure 1 presents the $T$ dependence of $2 \pi T D_{s}$ as calculated by lattice QCD, i.e., Banerjee (pink circles [32]), Kaczmarek (blue square [33]) and Ding (red triangle [34]), as well as the results modeled via the two approaches, i.e., Model-A

\footnotetext{
${ }^{1}$ Assuming an isotropic momentum dependence of the diffusion coefficient with the post-point scheme.
} 


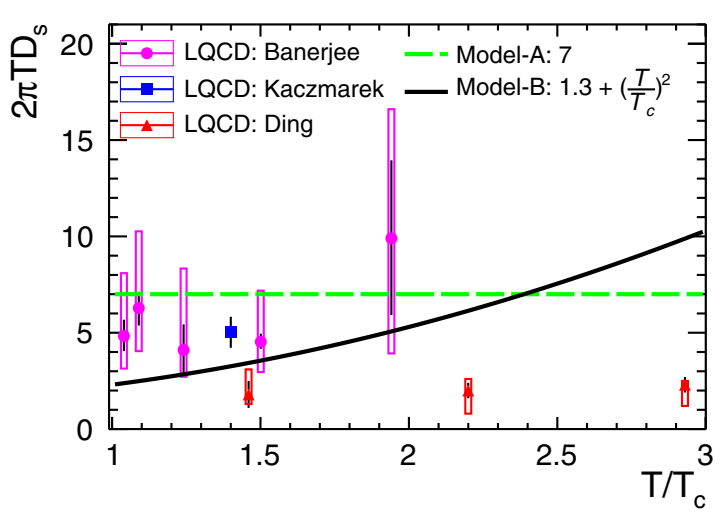

FIG. 1. Charm quark spatial diffusion coefficient $2 \pi T D_{s}$ calculated by lattice QCD at zero momentum: pink circle [32], blue square [33], and red triangle [34]. The phenomenological approaches (dashed green and solid black curves) are displayed as well.

[dashed green curve, Eq. (10)] and Model-B [solid black curve; Eq. (11)]. The corresponding results are summarized in Table I. It is found that most of the results obtained for the momentum diffusion coefficient $\kappa / T^{3}$ and HQ transport coefficient $\hat{q}_{Q} / T^{3}$ are consistent with the other model predictions within the significant systematic uncertainties.

For charm quarks, the relevant thermalization times defined in the zero momentum limit [30],

$$
\tau_{\text {charm }}=\frac{m_{\text {charm }}}{2 \pi T_{c}^{2}} \frac{2 \pi T D_{s}}{\left(T / T_{c}\right)^{2}},
$$

are 3.03 and $2.29 \mathrm{fm} / c$ for Model-A and Model-B, respectively, in $\mathrm{Pb}-\mathrm{Pb}$ collisions at 2.76 and $5.02 \mathrm{TeV}$ with $T=$ $2 T_{c}=330 \mathrm{MeV}$ and $m_{\text {charm }}=1.5 \mathrm{GeV}$.

Figure 2 shows the average in-medium energy loss of charm quarks as a function of the initial energy in central $(0-10 \%) \mathrm{Pb}-\mathrm{Pb}$ collisions at $\sqrt{s_{N N}}=5.02 \mathrm{TeV}$, displaying separately the contributions of collisional (long dashed blue curve) and radiative (dashed black curve) mechanisms. The results based on Model-A [Eq. (10)] are shown in the upper panel (a). As pointed out in Ref. [22], the collisional energy loss is significant at low energy, while radiative energy loss is the dominant mechanism at high energy. The crossing point between collisional and radiative contributions is around $E=7-8 \mathrm{GeV}$. Since the drag force is proportional to charm velocity $v=p / E$ [Eqs. (4) and (8)], in the low energy region $(E \lesssim 7-8 \mathrm{GeV})$, where the relativistic effect is trivial $(E \propto$

TABLE I. Summary of the different approaches for $2 \pi T D_{s}$ as a function of temperature (see Fig. 1), as well as the relevant results obtained for $\kappa / T^{3}$ and $\hat{q}_{Q} / T^{3}$. The other model predictions are shown for comparison.

\begin{tabular}{lccc}
\hline \hline & Model-A & Model-B & Reference \\
\hline $2 \pi T D_{s}$ & 7 & $1.3+\left(\frac{T}{T_{c}}\right)^{2}$ & \\
$\frac{\kappa}{T^{3}}\left(\frac{T}{T_{c}}=1.5\right)$ & 1.80 & 3.53 & $1.8-3.4[35]$ \\
$\frac{\hat{q}_{Q}}{T^{3}}\left(\frac{T}{T_{c}}=1.88\right)$ & 3.59 & 5.20 & $3.4-5.8[36]$ \\
$\frac{\hat{q}_{Q}}{T^{3}}\left(\frac{T}{T_{c}}=2.61\right)$ & 3.59 & 3.11 & $2.3-5.1[36]$ \\
\hline \hline
\end{tabular}
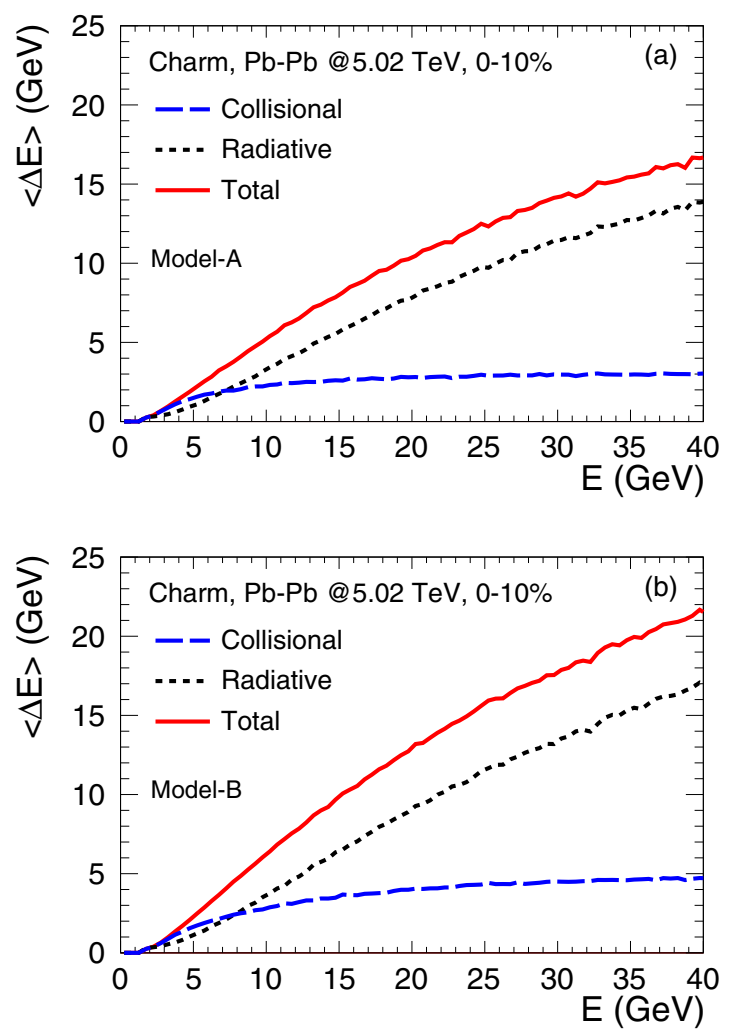

FIG. 2. Energy loss of charm quarks obtained via (a) Model-A and (b) Model-B; collisional and radiative contributions are shown separately as long-dashed blue and dashed black curves, respectively, in each panel. The combined results are shown as a solid red curve.

$v^{2}$ ), the collisional energy loss will be significant. However, in the very large energy region $(E \gtrsim 20 \mathrm{GeV})$, where the ultrarelativistic effect should be taken into account $(E \propto$ $1 / \sqrt{1-v^{2}}$ and $v \lesssim 1$ ), the collisional contribution will increase slowly at larger energy. In this case, the radiative energy loss will be the most dominant energy loss mechanisms. The results based on Model-B [Eq. (11)] are displayed in the bottom panel (b) of Fig. 2. A qualitatively similar trend can be found with Model-B, but with slightly stronger energy loss effects. This is caused by the facts that [22] (1) the underlying medium temperature drops rapidly from its initial value, and the charm quark will stay longer at low temperature $\left(\sim 1-2 T_{c}\right)$; (2) the initial transverse momentum spectrum of the charm quark is much harder than that of the medium constituent, thus the multiple elastic scatterings among them are dominated by the drag term rather than the diffusion term; (3) there is a larger drag coefficient near $T_{c}$ with Model$\mathrm{B}$, resulting in a stronger interaction strength between the injected charm quark and the incident medium constituents; consequently, the charm quark loses more of its energy with Model-B approach.

\section{Heavy quark hadronization}

When the local temperature is below the critical one $T_{c}=165 \mathrm{GeV}$, the charm quark will undergo instantaneous hadronization via a "dual" approach, including fragmentation 
TABLE II. Summary of the different fragmentation functions, as well as the relevant parameters. Note that $m_{\text {hadron }}$ and $m_{c}$ in Braaten are the masses of the open charmed hadron and its mother charm quark, respectively.

\begin{tabular}{lll}
\hline \hline \multicolumn{1}{c}{ Name } & \multicolumn{1}{c}{ Frag. function } & \multicolumn{1}{c}{ Parameter } \\
\hline Lund-PYTHIA & Eq. (12.11) in Ref. [37] & Default ones \\
Peterson & Eq. (4) in Ref. [38] & $\epsilon_{c}=0.06$ \\
Collins-Spiller & Eq. (20) in Ref. [39] & $\epsilon_{c}=0.01$ \\
Braaten & Eqs. (9) and (12) in Ref. [40] & $r=\frac{m_{\text {hadron }} m_{c}}{m_{\text {hadron }}}$ \\
FONLL-style & Eqs. (9) and (12) in Ref. [40] & $r=0.1[41]$ \\
\hline \hline
\end{tabular}

and heavy-light coalescence mechanisms. Concerning the universal fragmentation functions, various models are adopted in this work, e.g., Lund-PYTHIA 6.4 [37], Peterson [38], CollinsSpiller [39], Braaten [40] and FONLL-style [41], which are summarized in Table II. Apart from the Lund-PYTHIA, the fragmentation fractions for the various hadron species are $f\left(c \rightarrow D^{0}\right)=0.566, f\left(c \rightarrow D^{+}\right)=0.227, f\left(c \rightarrow D^{*+}\right)=$ 0.230 , and $f\left(c \rightarrow D_{s}^{+}\right)=0.081$ [22], respectively, in the other approaches.

According to the heavy-light coalescence model [42], the momentum distributions of heavy-flavor mesons $(Q \bar{q})$ are given as

$$
\begin{aligned}
\frac{d N_{M}}{d^{3} \vec{p}_{M}}= & g_{M} \int d^{3} \vec{x}_{Q} d^{3} \vec{p}_{Q} d^{3} \vec{x}_{\bar{q}} d^{3} \vec{p}_{\bar{q}} f_{Q}\left(\vec{x}_{Q}, \vec{p}_{Q}\right) f_{\bar{q}}\left(\vec{x}_{\bar{q}}, \vec{p}_{\bar{q}}\right) \\
& \times \bar{W}_{M}^{(n)}(\vec{y}, \vec{k}) \delta^{(3)}\left(\vec{p}_{M}-\vec{p}_{Q}-\vec{p}_{\bar{q}}\right),
\end{aligned}
$$

where $g_{M}$ is the degeneracy factor; $f_{Q}\left(\vec{x}_{Q}, \vec{p}_{Q}\right)$ and $f_{\bar{q}}\left(\vec{x}_{\bar{q}}, \vec{p}_{\bar{q}}\right)$ are the phase-space distributions of the heavy quark and light antiquark (i.e., the coalescence candidate), respectively. The coalescence probability for a $Q \bar{q}$ combination to form a heavy-flavor meson in the $n$th excited state is quantified by

$$
\bar{W}_{M}^{(n)}(\vec{y}, \vec{k})=\frac{v^{n}}{n !} e^{-v}, \quad v=\frac{1}{2}\left(\frac{\vec{y}^{2}}{\sigma_{M}^{2}}+\sigma_{M}^{2} \vec{k}^{2}\right),
$$

where

$$
\begin{aligned}
& \vec{y}=\vec{x}_{Q}-\vec{x}_{\bar{q}}, \\
& \vec{k}=\left(m_{\bar{q}} \vec{p}_{Q}-m_{Q} \vec{p}_{\bar{q}}\right) /\left(m_{Q}+m_{\bar{q}}\right),
\end{aligned}
$$

are the relative coordinate and the relative momentum, respectively, in the center-of-mass frame of the $Q \bar{q}$ pair. The width parameter $\sigma_{M}$ can be written as [22]

$$
\sigma_{M}^{2}= \begin{cases}\frac{2}{3} \frac{\left(e_{Q}+e_{\bar{q}}\right)\left(m_{Q}+m_{\bar{q}}\right)^{2}}{e_{Q} m_{\bar{q}}^{2}+e_{\bar{q}} m_{Q}^{2}}\left\langle r_{M}^{2}\right\rangle & (n=0), \\ \frac{2}{5} \frac{\left(e_{Q}+e_{\bar{q}}\right)\left(m_{Q}+m_{\bar{q}}\right)^{2}}{e_{Q} m_{\bar{q}}^{2}+e_{\bar{q}} m_{Q}^{2}}\left\langle r_{M}^{2}\right\rangle & (n=1),\end{cases}
$$

where $\left\langle r_{M}^{2}\right\rangle \approx(0.9 \mathrm{fm})^{2}$ is the mean-square charge radius of a $D$ meson; $e_{Q}$ and $e_{\bar{q}}$ are the absolute values of the charge of a heavy quark and a light antiquark, respectively; the light (anti)quark mass takes $m_{u / \bar{u}}=m_{d / \bar{d}}=300 \mathrm{MeV}$ and $m_{s / \bar{s}}=$ $475 \mathrm{MeV}$.

We consider the charm-strange meson species up to their first excited states $(n \leqslant 1)$, which are listed in detail in

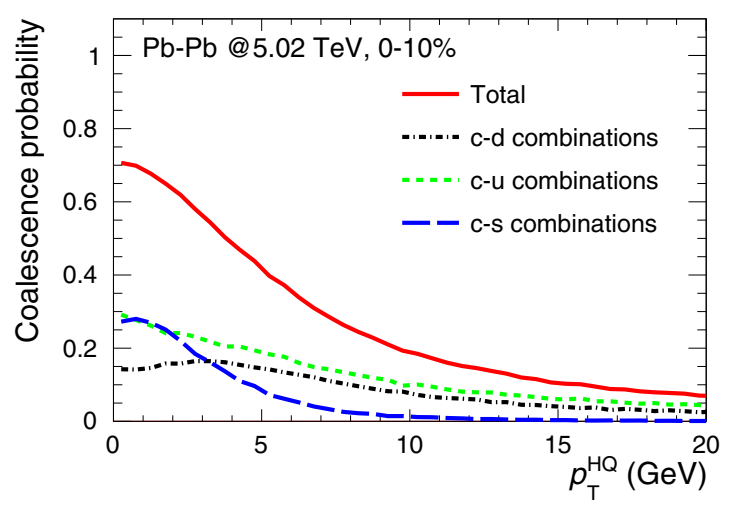

FIG. 3. Comparison of the coalescence probability contributed by different combinations in central $(0-10 \%) \mathrm{Pb}-\mathrm{Pb}$ collisions at $\sqrt{s_{N N}}=5.02 \mathrm{TeV}: c d$ (dot-dashed black curve), $c u$ (dashed green curve) and $c s$ (long-dashed blue curve). Both the ground states and the first excited states are considered. The combined results (solid red curve) are presented as well.

Ref. [22]. Figure 3 shows the coalescence probability obtained in central $(0-10 \%) \mathrm{Pb}-\mathrm{Pb}$ collisions at $\sqrt{s_{N N}}=5.02 \mathrm{TeV}$, as a function of the charm quark transverse momentum $\left(p_{T}\right)$. The results for the charm quark combined with a down quark $(c d)$, up quark $(c u)$, and strange quark (cs) are presented as dot-dashed black, dashed green and long-dashed blue curves, respectively. As shown in Eqs. (14) and (16), the quark mass and its charge play the role of the weighting factor in the heavy-light coalescence model, resulting in the difference among $c d, c u$, and $c s$ combinations. Moreover, this difference can also be induced at a certain amount by the thermal spectrum of $u / d$ and $s$ quarks, which is steeper for the former one, indicating a larger probability to sample the light quark with small $p_{T}$. Finally, it is found that the charm quark prefers to coalesce with $u$ and $s$ quarks in the range $p_{T} \lesssim 3 \mathrm{GeV}$. The total results (solid red curve) show a decreasing behavior with increasing $p_{T}$, varying from 0.7 at $p_{T} \sim 0$ to 0.2 at $p_{T} \sim$ $10 \mathrm{GeV}$, hence, the HQ with low/moderate and high $p_{T}$ tends to hadronize via coalescence and fragmentation mechanisms, respectively.

Note that the coalescence candidates are sampled among various light (anti)quarks, which are assumed to thermalize inside the QGP. Therefore, we utilize the Fermi-Dirac approach, $f_{q}(\vec{p}) \propto 1 / \exp \left\{\sqrt{\vec{p}^{2}+m_{q}^{2}} / T_{c}+1\right\}$, to describe its density distribution, where $m_{q}$ is the light (anti)quark mass and $T_{c}=$ $165 \mathrm{MeV}$ is the critical temperature. The flavor of the light (anti)quark is determined according to the integrated parton density $\rho=\int_{-\infty}^{\infty} d^{3} \vec{p} f_{q}(\vec{p})$. For instance, $\rho_{u / d}=0.18 \mathrm{fm}^{-3}$ for $u / \bar{u}$ and $d / \bar{d}$ quarks and $\rho_{s}=0.10 \mathrm{fm}^{-3}$ for $s / \bar{s}$ quarks, resulting in the relative ratio $\rho_{u}: \rho_{d}: \rho_{s} \approx 1: 1: 0.5$, which is kept during the sampling procedure.

\section{B. Theoretical uncertainty}

In this analysis, the total theoretical uncertainty consists of three components: FONLL predictions, nuclear shadowing and fragmentation models, which are added in quadrature for the final predictions. 


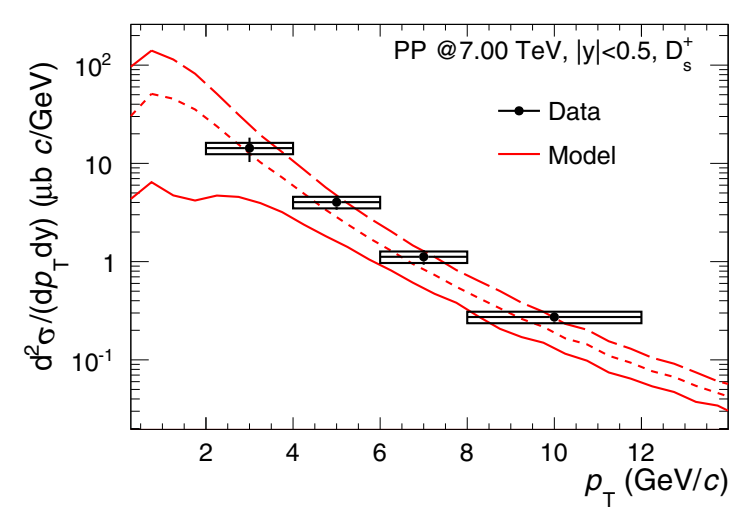

FIG. 4. $p_{T}$-differential production cross section of the $D_{s}^{+}$meson with $|y|<0.5$ in $p p$ collisions at $\sqrt{s}=7 \mathrm{TeV}$. Experimental data were taken from Ref. [45].

The initial charm quark spectra are determined by the FONLL calculations [24], as well as the corresponding central values obtained by setting $\mu_{R}=\mu_{F}=\mu_{0} \equiv \sqrt{p_{T}^{2}+m_{c}^{2}}$, where, $\mu_{R}\left(\mu_{F}\right)$ is the renormalization (factorization) scale; $m_{c}$ denotes the heavy quark mass, and its central value is $m_{c}=1.5 \mathrm{GeV}$. The relevant uncertainties are estimated via a conservative approach [43]: $\mu_{0} / 2<\mu_{R}, \mu_{F}<2 \mu_{0}, \mu_{R} / 2<$ $\mu_{F}<2 \mu_{R}$ and $1.3<m_{c}<1.7 \mathrm{GeV}$.

The uncertainty on nuclear shadowing is estimated according to the various nuclear parton distribution function (nPDF) sets in the EPS09NLO parametrization, which are obtained by tuning the fit parameters to reproduce the available measurements [27]. In this work, we employ the nPDF sets up to $k=7$. See Eqs. (2.12) and (2.13) in Ref. [27] for details.

Based on the different fragmentation scenarios (see Table II), the final observables such as the production cross section are close to each other. Therefore, we take the averaged results among them as the final one, and the maximum dispersion gives the theoretical uncertainty.

\section{RESULTS}

\section{A. Production cross section in $p p$ collisions}

In Fig. 4 the $p_{T}$-differential production cross section of the $D_{s}^{+}$meson is predicted at mid-rapidity $(|y|<0.5)$ in $p p$ collisions at $\sqrt{s}=7 \mathrm{TeV}$. The central value, the upper band, and the lower band are displayed as dashed, long-dashed, and solid curves, respectively. The uncertainty on FONLL calculations $(50-100 \%)$ is dominant at $2 \lesssim p_{T} \lesssim 8 \mathrm{GeV} / c$ compared with the one on fragmentation models $(\sim 30 \%$ at maximum), while they are compatible $(\sim 30 \%)$ toward larger $p_{T}$. The experimental data (boxes) are shown for comparison. Within the experimental and theoretical uncertainties, the measured $p_{T}$ dependence can be well described by the model predictions. A similar conclusion can be found in $p p$ collisions at $\sqrt{s}=5.02 \mathrm{TeV}^{2}$

\footnotetext{
${ }^{2}$ The $D_{s}^{+}$spectrum in $p p$ collisions at $\sqrt{s}=5.02 \mathrm{TeV}$ is obtained via $d \sigma_{p p} / d p_{T}=R_{A A} d \sigma_{A A} / d p_{T}$, while the corresponding $R_{A A}$ and $d \sigma_{A A} / d p_{T}$ are reported in Ref. [44].
}

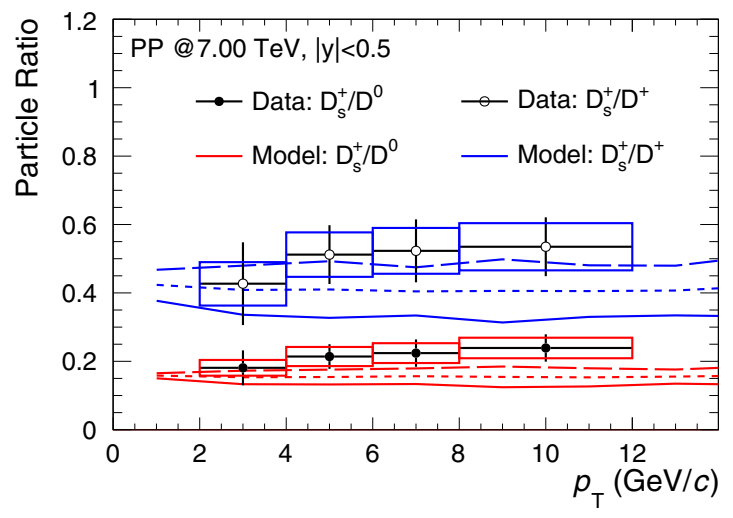

FIG. 5. Ratios of $D$-meson production cross sections as a function of $p_{T}$. the measurements for $D_{s}^{+} / D^{0}$ (solid) and $D_{s}^{+} / D^{+}$ (empty) are shown as boxes, while the relevant model predictions are displayed as the bands. Experimental data were taken from Ref. [45].

Figure 5 presents the ratios of the charm-strange meson $D^{+}$with respect to the nonstrange charmed mesons such as $D^{0}$ and $D^{+}$, in $p p$ collisions at $\sqrt{s}=7 \mathrm{TeV}$. See the legend for details. For both $D_{s}^{+} / D^{0}$ and $D_{s}^{+} / D^{+}$, the theoretical uncertainty on FONLL calculations $(\sim 10 \%$ at maximum) is dominant in the range $2 \lesssim p_{T} \lesssim 4 \mathrm{GeV} / c$, while the one on fragmentation models $(\sim 20 \%$ at maximum) is dominant at higher $p_{T}$. It is found that, within uncertainties, the measurements can be reproduced by the corresponding model predictions.

\section{B. Nuclear modification factor and elliptic flow}

Panel (a) of Fig. 6 shows the average $R_{A A}$ of nonstrange charmed mesons $\left(D^{0}, D^{+}\right.$, and $\left.D^{*+}\right)$ at mid-rapidity $(|y|<0.5)$, with the Model-A approach [Eq. (10)] in central $(0-10 \%) \mathrm{Pb}-\mathrm{Pb}$ collisions at $\sqrt{s_{N N}}=5.02 \mathrm{TeV}$, which is contributed by the various hadronizaton mechanisms. It is found that the fragmentation component (long dashed black curve) is dominant at $p_{T} \gtrsim 7-8 \mathrm{GeV} / c$, while the coalescence (dotted green curve) is significant at $1 \lesssim p_{T} \lesssim 5 \mathrm{GeV} / c$, and furthermore the first excited states contribution (dot-dashed purple curve) is more pronounced in this region. The central prediction (solid red curve) can describe the measurement in the range $p_{T}>5 \mathrm{GeV} / c . R_{A A}$ of the charm-strange meson $\left(D_{s}^{+}\right)$is presented in panel (b) of Fig. 6. Similar behavior is observed when comparing with the nonstrange charmed mesons; however, the coalescence effect is more pronounced for the $D_{s}^{+}$meson. It is further checked that $R_{A A}$ (average) and $R_{A A}\left(D_{s}^{+}\right)$, calculated by considering alone the fragmentation mechanism, are close to each other. All the conclusions drawn above are the same as the ones found in semicentral (30-50\%) $\mathrm{Pb}-\mathrm{Pb}$ collisions at $\sqrt{s_{N N}}=5.02 \mathrm{TeV}$, as well as in $\mathrm{Pb}-\mathrm{Pb}$ collisions at $\sqrt{s_{N N}}=2.76 \mathrm{TeV}$. Therefore, future measurements of $R_{A A}\left(D_{s}^{+}\right)$with higher precision will more effectively constrain the heavy-light coalescence effect at moderate $p_{T}$ $\left(p_{T}=2-5 \mathrm{GeV} / c\right)$.

$R_{A A}$ (average) (solid curves) and $R_{A A}\left(D_{s}^{+}\right)$(dashed curves) obtained at mid-rapidity $(|y|<0.5)$, with the Model-A approach in central $(0-10 \%) \mathrm{Pb}-\mathrm{Pb}$ collisions at 

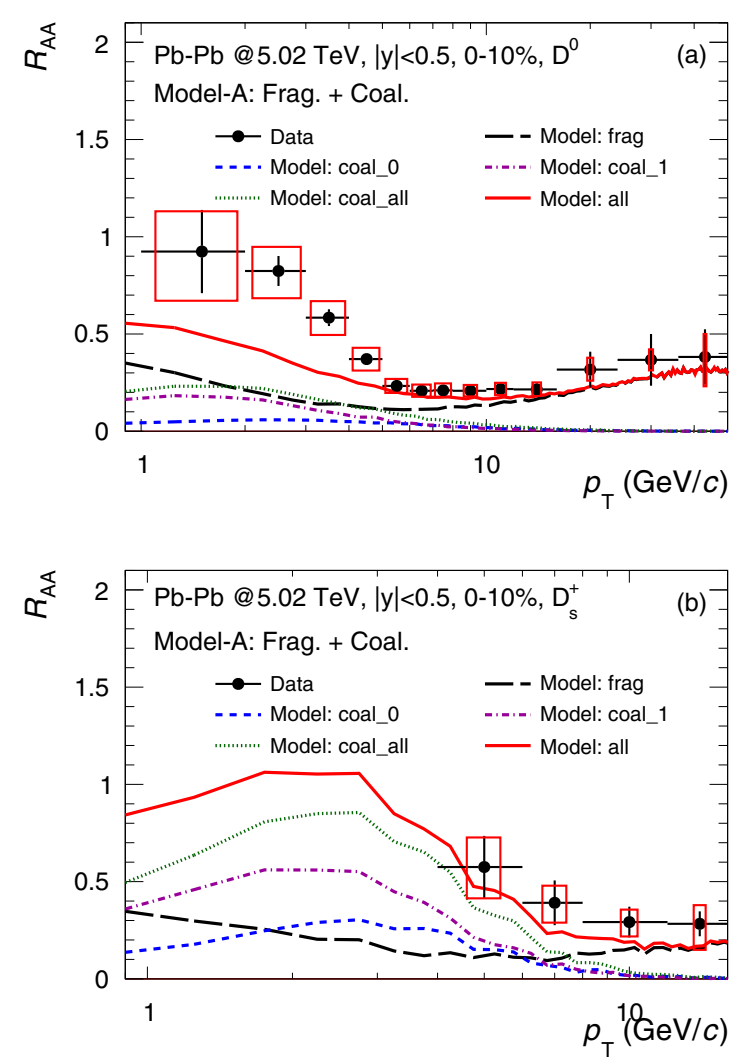

FIG. 6. Comparison of the central predictions of $R_{A A}$ contributed by different hadronization mechanisms: for (a) the nonstrange charmed mesons (average among $D^{0}, D^{+}$, and $D^{*+}$ ) and (b) the charm-strange meson $\left(D_{s}^{+}\right)$, at mid-rapidity $(|y|<0.5)$ in central $(0-10 \%) \mathrm{Pb}-\mathrm{Pb}$ collisions at $\sqrt{s_{N N}}=5.02 \mathrm{TeV}$. See legend for details. Experimental data were taken from Ref. [44].

$\sqrt{s_{N N}}=5.02 \mathrm{TeV}$, are shown in panel (a) of Fig. 7. The results between the average of nonstrange $D$ mesons and $D_{s}^{+}$are similar in the range $p_{T} \gtrsim 6 \mathrm{GeV} / c\left(p_{T} \gg m_{c}\right)$, while the latter one is systematically larger at $2<p_{T}<5 \mathrm{GeV} / c$, resulting in an enhancement of $D_{s}^{+}$production with respect to the average one. As mentioned in Fig. 6, the enhancement behavior is mainly induced by the coalescence mechanism during the charm quark hadronization. Note that, for $R_{A A}$ (average), the uncertainty on FONLL calculations $(\sim 80 \%$ at maximum) is dominant at $1<p_{T}<3 \mathrm{GeV} / c$, while the ones on nuclear shadowing $(\lesssim 10 \%)$ and fragmentation functions $(\sim 10-15 \%)$ are significant at higher $p_{T}$. Similar behavior can be found for $R_{A A}\left(D_{s}^{+}\right)$at low $p_{T}$, but the uncertainty on fragmentation functions $(\sim 20-40 \%)$ is dominant at $p_{T}>3 \mathrm{GeV} / c$. For comparison, the available measurements for the average of nonstrange $D$ mesons (solid) and the $D_{s}^{+}$meson (empty) are displayed as well. Within the experimental and theoretical uncertainties, the model calculations can reproduce the data for both the average and the $D_{s}^{+}$meson. Similar results are found in central $(0-10 \%) \mathrm{Pb}-\mathrm{Pb}$ collisions at $\sqrt{s_{N N}}=2.76 \mathrm{TeV}$. See panel (b) of Fig. 7 for details.

To compare the predictions based on Model-A [Eq. (10)] and Model-B approaches [Eq. (11)], panel (a) of Fig. 8 presents $R_{A A}$ (average) and $R_{A A}\left(D_{s}^{+}\right)$calculated in
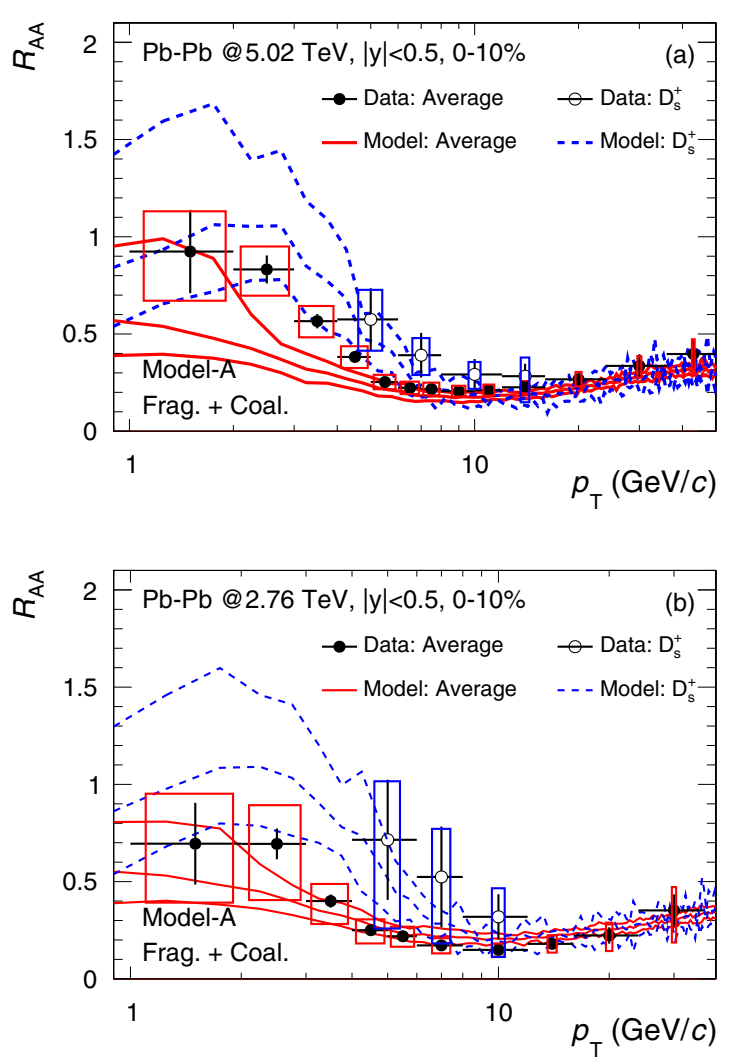

FIG. 7. Comparison of $R_{A A}$ for the average of nonstrange $D$ mesons (solid red curves) and $D_{s}^{+}$(dashed blue curves) predicted at mid-rapidity $(|y|<0.5)$ in central $(0-10 \%) \mathrm{Pb}-\mathrm{Pb}$ collisions at (a) $\sqrt{s_{N N}}=5.02 \mathrm{TeV}$ and (b) $2.76 \mathrm{TeV}$, respectively. Experimental data were taken from Ref. [44,46].

central $(0-10 \%) \mathrm{Pb}-\mathrm{Pb}$ collisions at $\sqrt{s_{N N}}=5.02 \mathrm{TeV}$. $R_{A A}$ (average) is enhanced (suppressed) at low (high) $p_{T}$ from Model-A (dot-dashed black curve) to Model-B (solid black curve); since the relevant $2 \pi T D_{s}$ near $T_{c}$ is smaller based on Model-B, the larger is its initial drag term, which is more powerful to pull $c \bar{c}$ pairs from high momentum to low momentum [22], as pointed in Sec. II A 2. The results between different models are close at $p_{T} \sim 2 \mathrm{GeV} / c$. Similar behavior is observed for $R_{A A}\left(D_{s}^{+}\right)$. When comparing $R_{A A}$ (average) with $R_{A A}\left(D_{S}^{+}\right)$, the results with the Model-A approach were discussed already in panel (a) of Fig. 7, and the same conclusion can be drawn with the Model-B approach. The panel (b) of Fig. 8 shows the elliptic flow coefficient $v_{2}$ predicted in semicentral $(30-50 \%) \mathrm{Pb}-\mathrm{Pb}$ collisions at $\sqrt{s_{N N}}=5.02 \mathrm{TeV}$ : both $v_{2}$ (average) and $v_{2}\left(D_{s}^{+}\right)$are significantly enhanced at intermediate $p_{T}$ $\left(2 \lesssim p_{T} \lesssim 4 \mathrm{GeV} / c\right)$ from Model-A (dot-dashed black curve) to Model-B (solid black curve). Performing the model-to-data comparison, it is realized that $R_{A A}$ (average) and $R_{A A}\left(D_{s}^{+}\right)$favor Model-A to have a better description of the measured $p_{T}$ dependence, while their $v_{2}$ prefer Model-B at moderate $p_{T}\left(2 \lesssim p_{T} \lesssim 4 \mathrm{GeV} / c\right)$, indicating the necessity to consider the temperature and/or momentum dependence of $2 \pi T D_{s}$ to describe simultaneously $R_{A A}$ and $v_{2}$ for both the nonstrange $D$ mesons and the $D_{s}^{+}$meson. 

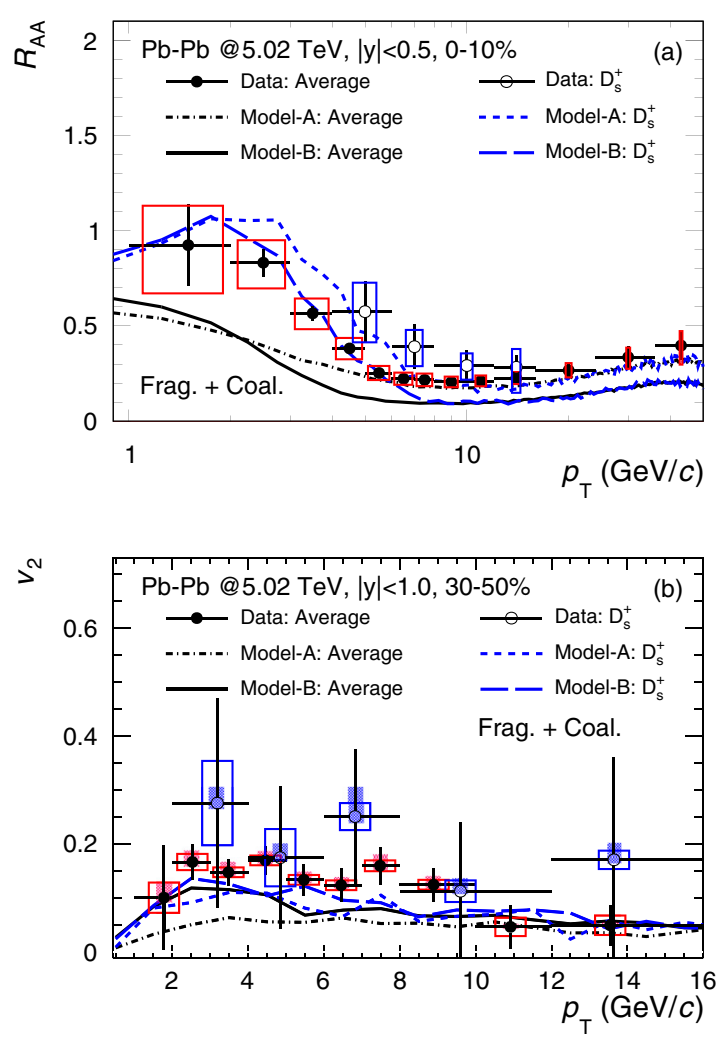

FIG. 8. Comparison between the average of nonstrange $D$ mesons and $D_{s}^{+}$observables obtained with Model-A and Model-B approaches in $\mathrm{Pb}-\mathrm{Pb}$ collisions at $\sqrt{s_{N N}}=5.02 \mathrm{TeV}: R_{A A}$ (upper) in $0-10 \%$ and $v_{2}$ in 30-50\%. Experimental data were taken from Refs. [44,47].

\section{SUMMARY AND CONCLUSIONS}

In this analysis, we aim to investigate the nuclear modification of $D_{s}^{+}$meson spectra together with its elliptic flow in ultrarelativistic heavy-ion collisions. We utilize the theoretical framework built in our previous work to achieve this goal, and extend it to include further the theoretical uncertainty on initial charm quark spectra, nuclear shadowing, and the fragmentation model. The coupling strength for the charm quark, $2 \pi T D_{s}$, is obtained by fitting lattice QCD calculations: $2 \pi T D_{s}=$ const (Model-A, i.e., no temperature dependence) and $2 \pi T D_{s}=1.3+\left(T / T_{c}\right)^{2}$ (Model-B, i.e., weak temperature dependence).

It is found that $D_{s}^{+}$spectra measured at mid-rapidity $(|y|<0.5)$ can be well described by the relevant model predictions in $p p$ collisions both at $\sqrt{s}=7$ and $5.02 \mathrm{TeV}$, as well as the derived particle ratios $D_{s}^{+} / D^{0}$ and $D_{s}^{+} / D^{+}$. The nuclear modification factor $R_{A A}\left(D_{s}^{+}\right)$is systematically larger than $R_{A A}$ (nonstrange) at intermediate $p_{T}\left(2 \lesssim p_{T} \lesssim 5 \mathrm{GeV}\right)$ in central $(0-10 \%)$ and semicentral $(30-50 \%) \mathrm{Pb}-\mathrm{Pb}$ collisions both at $\sqrt{s_{N N}}=5.02$ and $2.76 \mathrm{TeV}$, which is mainly induced by the heavy-light coalescence mechanism. Hence, future measurements of $R_{A A}\left(D_{s}^{+}\right)$with higher precision will more effectively constrain the heavy-light coalescence effect at moderate $p_{T}\left(p_{T}=2-5 \mathrm{GeV} / c\right)$. For the model-to-data comparisons, the predictions of $R_{A A}\left(D_{S}^{+}\right)$and $R_{A A}$ (nonstrange) favor Model-A to reproduce well the measured $p_{T}$ dependence in both colliding energies, while they $v_{2}$ prefer Model-B at moderate $p_{T}\left(2 \lesssim p_{T} \lesssim 4 \mathrm{GeV}\right)$, suggesting a temperature and/or momentum dependent $2 \pi T D_{s}$ is needed to describe simultaneously the $D$-meson $R_{A A}$ and $v_{2}$ data.

\section{ACKNOWLEDGMENTS}

The authors are grateful to Kejun Wu and Kyong Chol Han for useful discussions. S.L. is supported by China Three Gorges University (CTGU) Contracts No. 1910103, Hubei Province Contracts No. B2018023, Key Laboratory of Quark and Lepton Physics Contracts No. QLPL2018P01, and National Science Foundation of China (NSFC) under Grant Nos. 11447023 and 11875178 . C.W.W. acknowledges support from National Science Foundation of Hubei Province (NSFHB) Contract. No. 2012FFA085.
[1] M. Gyulassy and L. McLerran, Nucl. Phys. A 750, 30 (2005).

[2] E. Shuryak, Nucl. Phys. A 750, 64 (2005).

[3] F. Prino and R. Rapp, J. Phys. G 43, 093002 (2016).

[4] J. Schukraft, Nucl. Phys. A 967, 1 (2017).

[5] R. Rapp et al., arXiv:1803.03824.

[6] S. Wicks, W. Horowitz, M. Djordjevic, and M. Gyulassy, Nucl. Phys. A 784, 426 (2007).

[7] M. Gyulassy and X. N. Wang, Nucl. Phys. B 420, 583 (1994).

[8] S. Li (for the ALICE Collaboration), Nucl. Phys. A 931, 546 (2014).

[9] J. Liao and E. Shuryak, Phys. Rev. C 75, 054907 (2007).

[10] H. van Hees, M. Mannarelli, V. Greco, and R. Rapp, Phys. Rev. Lett. 100, 192301 (2008).

[11] S. Cao, G. Y. Qin, and S. A. Bass, Phys. Rev. C 88, 044907 (2013).

[12] M. Nahrgang, J. Aichelin, P. B. Gossiaux, and K. Werner, Phys. Rev. C 93, 044909 (2016).

[13] T. Song, H. Berrehrah, D. Cabrera, W. Cassing, and E. Bratkovskaya, Phys. Rev. C 93, 034906 (2016).
[14] A. Beraudo, A. D. Pace, M. Monteno, M. Nardi, and F. Prino, J. High Energy Phys. 02 (2018) 043.

[15] J. F. Liao and E. Shuryak, Phys. Rev. Lett. 102, 202302 (2009).

[16] J. C. Xu, J. F. Liao, and M. Gyulassy, Chin. Phys. Lett. 32, 092501 (2015).

[17] S. K. Das, F. Scardina, S. Plumari, and V. Greco, Phys. Lett. B 747, 260 (2015).

[18] J. C. Xu, J. F. Liao, and M. Gyulassy, J. High Energy Phys. 02 (2016) 169.

[19] J. Rafelski and B. Müller, Phys. Rev. Lett 48, 1066 (1982).

[20] M. He, R. J. Fries, and R. Rapp, Phys. Rev. Lett. 110, 112301 (2013).

[21] G. Aarts et al., Eur. Phys. J. A 54, 93 (2017).

[22] S. Li, C. W. Wang, X. B. Yuan, and S. Q. Feng, Phys. Rev. C 98, 014909 (2018).

[23] C. Shen, Z. Qiu, H. C. Song, J. Bernhard, S. A. Bass, and U. Heinz, Comput. Phys. Commun. 199, 61 (2016).

[24] M. Cacciari, M. Greco, and P. Nason, J. High Energy Phys. 05 (1998) 007. 
[25] M. Cacciari, S. Frixione, and P. Nason, J. High Energy Phys. 03 (2001) 006.

[26] M. Cacciari, S. Frixione, N. Houdeau, M. L. Mangano, P. Nason, and G. Ridolfi, J. High Energy Phys. 10 (2012) 137.

[27] K. J. Eskola, H. Paukkunen, and C. A. Salgado, J. High Energy Phys. 04 (2009) 065.

[28] I. Karpenko, P. Huovinen, and M. Bleicher, Comput. Phys. Commun. 185, 3016 (2014).

[29] B. W. Zhang, E. K. Wang, and X. N. Wang, Phys. Rev. Lett. 93, 072301 (2004).

[30] G. D. Moore and D. Teaney, Phys. Rev. C 71, 064904 (2005).

[31] V. Greco, J. Phys. Conf. Ser. 779, 012022 (2017).

[32] D. Banerjee, S. Datta, R. Gavai, and P. Majumdar, Phys. Rev. D 85, 014510 (2012).

[33] O. Kaczmarek, Nucl. Phys. A 931, 633 (2014).

[34] H. T. Ding, A. Francis, O. Kaczmarek, F. Karsch, H. Satz, and W. Soeldner, Phys. Rev. D 86, 014509 (2012).

[35] A. Francis, O. Kaczmarek, M. Laine, T. Neuhaus, and H. Ohno, Phys. Rev. D 92, 116003 (2015).

[36] JET Collaboration, Phys. Rev. C 90, 014909 (2014).
[37] T. Sjöstrand, S. Mrenna, and P. Z. Skands, J. High Energy Phys. 05 (2006) 026.

[38] C. Peterson, D. Schlatter, I. Schmitt, and P. M. Zerwas, Phys. Rev. D 27, 105 (1983).

[39] P. D. B. Collins and T. P. Spiller, J. Phys. G 11, 1289 (1985).

[40] E. Braaten, K. Cheung, and T. C. Yuan, Phys. Rev. D 48, R5049 (1993).

[41] M. Cacciari, P. Nason, and R. Vogt, Phys. Rev. Lett. 95, 122001 (2005).

[42] K. C. Han, R. J. Fries, and C. M. Ko, Phys. Rev. C 93, 045207 (2016).

[43] R. Averbeck, N. Bastid, Z. Conesa del Valle, P. Crochet, A. Dainese, and X. Zhang, arXiv:1107.3243.

[44] S. Acharya et al. (ALICE Collaboration), arXiv:1804.09083.

[45] G. M. Innocenti (for the ALICE Collaboration), Eur. Phys. J. C 77, 550 (2017).

[46] J. Adam et al. (ALICE Collaboration), J. High Energy Phys. 03 (2016) 081.

[47] S. Acharya et al. (ALICE Collaboration), Phys. Rev. Lett. 120, 102301 (2018). 